\title{
El primer año de los estudios universitarios: análisis de una propuesta tutorial para alumnos de la carrera de economía de la Universidad Nacional del Sur
}

The First Year of University Studies: Analysis of a Tutorial Proposal for Students of Economics at Universidad Nacional del Sur Primeiro ano de estudos universitários: análise de uma proposta tutorial para estudantes de Economia da Universidad Nacional del Sur

Diana Isabela Lis

Universidad Nacional del Sur, Argentina

E-mail:dlis@uns.edu.ar

Carolina Tarayre

Universidad Nacional del Sur, Argentina

E-mail: carotarayre@gmail.com

\author{
Déborah Malisani \\ Universidad Nacional del Sur, Argentina \\ E-mail:debymalisani@gmail.com
}

Fecha de Recepción:02/03/2021 Fecha de Aceptación: 22/09/2021

\section{Resumen}

En los últimos años, las tutorías enfocadas en alumnos ingresantes de los primeros años de las carreras universitarias han ido ganando reconocimiento por ser consideradas relevantes en el pasaje del nivel secundario al superior.

En este trabajo se describe y evalúa una acción tutorial asociada a la función docente para ingresantes de las carreras Licenciatura en Economía y Profesorado en Economía pertenecientes al Departamento de Economía de la Universidad Nacional del Sur (UNS), Argentina, cuyos resultados invitan a avanzar hacia un modelo integral de tutorías, sin desconocer las cuestiones que pueden obturar este objetivo.

Palabras clave

- ingreso

- universidad

- articulación

- taller

- análisis
En principio, se realiza la caracterización de las Tutorías del Departamento de Economía de la UNS para luego mencionar los lineamientos conceptuales base que son sustento de la acción tutorial específica.

Más adelante, se realiza el relato de la experiencia: Taller de Estrategias de Aprendizaje. También, se describe la metodología y análisis que se 
utilizó para lograr su evaluación y finalmente se arriba a las reflexiones que invitan a pensar en acciones tutoriales situadas y flexibles que faciliten la trayectoria de los ingresantes universitarios.

\begin{abstract}
In recent years, tutorships aimed at students in the first years of their university studies have been acknowledged as relevant in the transition from secondary to higher level education.

This article describes and evaluates a tutorship for first-year students of the Bachelor and Professorship in Economics programmes, which belong to the Department of Economics of Universidad Nacional del Sur (UNS), Argentina.

First, the tutorship programmes of the Tutorship Department of Economics, UNS, are described; then their main conceptual guidelines are pointed out. An account of the experience "Learning Strategies Workshop" is provided later.

In addition, the methodology and analysis used to achieve this evaluation are described. Finally, from the reflection upon the experience, some considerations related to further tutorship programmes that could help first-year university students in this transition are provided.
\end{abstract}

Keywords

- admission

- analysis

- articulation

- university

- workshop

Palavras-chave

- análise

- articulação

- ingresso

- oficina

- universidade

\title{
Resumo
}

Nos últimos anos, os programas de assistência estudantil destinados aos estudantes universitários do primeiro ano, têm ganhado reconhecimento por serem considerados relevantes na transição do nível secundário para 0 superior.

Neste artigo se descreve e avalia uma ação de assistência relacionada à função docente para ingressantes dos cursos de Bacharelado $\mathrm{e}$ Licenciatura em Economia, pertencentes ao Departamento de Economia da Universidade Nacional del Sul (UNS), cujos resultados convidam a avançar para um modelo integral de tutorias, sem desconhecer questões que possam obturar este objetivo.

Em princípio, apresentamos uma caracterização do programa de assistência do Departamento de Economia da UNS para mencionar posteriormente as diretrizes conceituais básicas que são alicerce da ação tutorial específica.

Posteriormente, realizamos um relato da experiência: Oficina de Estratégias da Aprendizagem. Além disso, descrevemos a metodologia e análise que foram utilizadas para realizar sua avaliação e finalmente chegar às reflexões que convidam o leitor a pensar em ações de assistência situadas e flexíveis que facilitem a trajetória dos ingressantes na universidade. 